\title{
Nonstandard topological extensions
}

\section{Robert A. Herrmann}

\begin{abstract}
This paper investigates the nonstandard theory of filters on a non-empty meet-semi-lattice of sets and applies this theory to the general study of topological extensions $Y$ for a space $X$. In particular, we apply this theory to Baire and quasi-H-closed extensions as well as Wallman type compactifications. Whereas these extensions have previously been obtained and studied as types of ultrafilter extensions, we study them as subsets of an enlargement of $X$. Since $X \subset Y \subset \hat{X}$ and the elements of $X$ and $Y-X$ are of the same set-theoretic type, these extensions appear more natural from the nonstandard viewpoint.
\end{abstract}

\section{Introduction}

Extensions of a space $X$ by means of filter type spaces have been studied by numerous authors. For example, the Wallman type compactifications using normal bases as introduced by Frink [5] and studied by Alo and Shapiro [1], Alò and Shapiro [2], Biles [3], D'Aristotle [4], Steiner and Steiner [17], Steiner [18], [19], among others. As shown in [6], the Stone-Čech compactification may be considered as a Z-ultrafilter extension. The Katétov extension of a Hausdorff space [9], the quasi- $H-$ closed (that is, generalized absolutely closed) extension such as it is discussed by Porter and Thomas [14] and Liu [10], and a Baire extension discovered by McCoy [13] are studied as ultrafilter type extensions determined by the topology of $X$.

At the Seventy-Ninth Annual Meeting of the American Mathematical Society I conjectured that all of the above extensions and many new ones could be obtained as subsets of an enlargement of $X,[7]$. I was asked to

Received 2 July 1975. 
justify this conjecture. Consequently, this paper investigates a construction method which shows that all of the above extensions, as well as many others, can be considered as subsets of $\hat{X}$, where $\hat{X}$ is the nonstandard extension of a space $X$ within the set-theoretic enlargement ${ }^{*} M=\left({ }^{*} U,{ }^{*} \varepsilon,{ }^{*} p r,{ }^{*} a p\right)$ discovered by Machover and Hirschfeld [12]. The extensions $Y$ of $X$ constructed here not only have the property that $X \subset Y \subset \hat{X}$ but they also appear more natural from the nonstandard viewpoint since the elements of $X$ and $Y-X$ are of the same set-theoretic type; whereas, in many of the classically constructed extensions the elements of the remainder are filters on $X$. Indeed, observe that if $q \in \hat{X}-X$, then $q$ is not an element of any $n$-fold power set iteration, $n>1$.

First, we show how it is possible to extend most of the known results from the nonstandard theory of filters on a set $X$ to filters in any nonempty meet-semi-lattice of subsets of $X$. We then use the topology or a normal base on a space $X$ as the basic meet-semi-lattice in order to construct various topological extensions as subsets of $\hat{X}$. Even though the meet-semi-lattices we use in the latter parts of this paper are lattices of sets, I have shown elsewhere [8] that other interesting extensions exist where the basic meet-semi-lattice $V$ is the set of all regular-open subsets of $X$. In this latter case, $V$ is known to be a non-lattice.

\section{2. $v-f i l$ ters}

Throughout this paper $L$ will be a suitable first order language with equality containing one binary predicate symbol " $\varepsilon$ " and two binary operator symbols "pr" and "ap" . We assume that the reader is familiar with the concepts and notations of set-theoretic nonstandard analysis the foundations of which may be found in references [11], [12], [15]. Our principal reference is [12] and the first order structure we use is $M=(U, \varepsilon, p r, a p)$ which is to be interpreted in the usual manner. We let our nonstandard structure be the enlargement ${ }^{*} M=\left({ }^{*} U,{ }^{*} \varepsilon,{ }^{*} p r,{ }^{*} a p\right)$. Any notation not specifically defined in this paper will be found in [12]. of course, we assume that for a topological space $(X, \tau)$, we have $X \in U$, where $U$ is our universe.

Let $V$ be any non-empty meet-semi-lattice of subsets of a non-empty $X$. This means that if $G, H \in V$, then $G \cap H \in V$. In the usual manner [16], we call any non-empty $F \subset V$ a $V$-filter if whenever $G, H \in F$, 
then $G \cap H \in F$ and whenever $A \in F, B \in V, A \subset B$, then $B \in F$. We say that a non-empty $F \subset V$ is a subbase for a $V$-filter $\langle F\rangle$ if $\langle F\rangle=\left\{p \mid[p \in V] \wedge \exists y\left[\left[y \in F^{\prime}\right] \wedge[y<p]\right]\right\}$, where $F^{\prime}=\left\{p \mid\left[p=\cap\left\{A_{i}\right\}\right] \wedge\left[\left\{A_{i}\right\}\right.\right.$ is a non-empty finite subset of $\left.\left.F\right]\right\}$. A $V$-filter $F$ is trivial if $F=V$. Finally, $\emptyset \neq B \subset V$ is a base for the $V$-filter $\langle B\rangle$ if for each $G, H \in B$ there exists some $K \in B$ such that $K \subset G \cap H$. Whenever we discuss a $V$-filter $F$, we will always tacitly assume that $\emptyset \neq F \subset V \subset P(X)$ and that $V$ is a meet-semi-lattice unless otherwise indicated.

The following results are easily verified.

(2.1). If $F$ is a V-filter and $\emptyset \neq B \subset F$, then $B$ is a base for $F$ iff for each $G \in F$ there exists some $H \in B$ such that $H \subset G$.

(2.2). If we assume that $\emptyset \in V$ and $\emptyset \neq F \subset V$, then $\langle F\rangle \neq V$ iff $F$ has the finite intersection property.

We call a U-filter $F$ a V-ultrafizter if it is a maximal ( $\subset$ ) non-trivial $v$-filter.

(2.3). If $\emptyset \in V$, then every non-trivial $V$-fizter is contained in a V-ultrafilter.

(2.4). If $\varnothing, G \in V$ and $F$ is a V-ultrafizter, then $G \cap H \neq \emptyset$ for each $H \in F$ iff $G \in F$.

(2.5). If $G, H \in V, G \cup H \in F$, where $F$ is a V-uztrafizter and $V$ is a lattice of subsets of $X$, then either $G \in F$ or $H \in F$.

We now indicate how to modify most of the known results from the nonstandard theory of filters on a set $X,[11],[12]$, so that they hold for $v$-filters. In order to do this, we formally prove only a small number of results and then state a representative collection of theorems, without proof, since the reader should be able to easily modify the corresponding filter results as they appear in [11], [12]. If it should become necessary to distinguish between various meet-semi-lattices we will use the terminology $V_{\text {-filter on } X}$ to mean that $U \subset P(X)$. Also, certain variants to the notation and terminology found in [12] will be introduced.

THEOREM 2.6. If $\emptyset \neq F \subset V$, then Nuc $F=$ Nuc $\langle F\rangle$.

Proof. Clearly $F \subset\langle F\rangle \subset G$, where $G$ is the filter on $X$ 
generated by $F$. From the definition of the "nucleus" (that is, Nuc $F=n\{\hat{G} \mid G \in F\}$ ), it follows that Nuc $G \subset$ Nuc $\langle F\rangle \subset$ Nuc $F$. Using the known result that Nuc $F=$ Nuc $G$, we have the result.

DEFINITION 2.1. Let $\varnothing \neq F \subset V$. Then Nuc $F=n\{\hat{F} \mid F \in F\}$ will be called a $V$-monad.

We observe, at this point, that if $F \subset V$ is a base for a $V$-filter, then it is also a base for a filter on $X$.

THEOREM 2.7. If $F$ is a V-fizter and $F_{1} \subset F$, then $F_{1}$ is a base for $F$ iff $F$ *contains an infinitesimal *element which is also a *element of $F_{1}$.

Proof. Let $F_{1} \subset F$ and assume that $F_{1}$ is a base for $F$. Then $F_{1}$ is a base for the filter $G$ on $X$ generated by $F_{I}$. Consequently, there exists some $E \in *^{*}$ such that $\hat{E} \subset$ Nuc $F_{1}=$ Nuc $G$ and $E * \in F_{1}$. Since $F_{1} \subset F$ implies that $\hat{F}_{1} \subset \hat{F}$, then $E * \in F$.

Conversely, assume that $F_{1} \subset F \subset V$ and that there exists some $E \in * U$ such that $E * \in F_{1}$ and $E$ is an infinitesimal *element of $F$. Hence, if $G \in F$, then $\hat{E} \subset \hat{G}$. Thus the sentence in $L$,

$$
\exists x\left[\left[x \in F_{1}\right] \wedge[x \subset G]\right]
$$

holds in $* M$; hence in $M$. Interpreting this in $M$, it follows that each element in $F$ contains a subset which is an element of $F_{1}$ and, of course, since $F_{I} \subset V$ also an element of $V$. This implies that $F_{1}$ is a base for the $V$-filter $F$.

DEFINITION 2.2. Let $W \subset \hat{X}$. Define

$$
V \text { Fil }(W)=\{G \mid[G \in V] \wedge[W \subset \hat{G}]\} \text {. }
$$

If $V$ Fil $(W) \neq \emptyset$, then clearly $V$ Fil $(W)$ is a $V$-filter on $X$. If we now replace the word "filter" by "V-filter" and the symbol "Fil" by "V Fil", then by similar modifications as displayed above it can easily be shown that Theorems 5.1.3 and 5.1.4 of [12] hold. Indeed, the following is also easily verified. 
THEOREM 2.8. Let $\langle F\rangle,\langle G\rangle$ be $V$-filters generated by bases $F$ and $G$. Then $\langle F\rangle \subset\langle G\rangle$ iff Nuc $G \subset$ Nuc $F$ iff for each $F \in F$ there exists some $G \in G$ such that $G \subset F$.

Now let $\left\{F_{j} \mid j \in J\right\}$ be a non-empty set of $V_{\text {-filters such that }}$ $n\left\{F_{j} \mid j \in J\right\}=H \neq \emptyset$. Clearly $H$ is the strongest (in the usual sense) $V$-filter weaker than each $F_{j}$. Under the above assumption, it follows that the results of Sections 5.1.6 and 5.1.7 of [12] hold when interpreted for $V_{\text {-filters. }}$

Let $B$ be a base for a $V$-filter $F$ on $X$ and $f: X \rightarrow Y$. Consider

$$
v_{y}=\left\{p \mid\left[p=n\left\{f\left[G_{i}\right]\right\}\right] \wedge\left[\left\{G_{i}\right\} \text { is a non-empty finite subset of } v\right]\right\} \text {. }
$$

Then it is not difficult to show that $f[B]$ is a base for the $v_{y}$-filter $(f[F])_{y}$ on $Y$. Also, if $\mathcal{C}$ is a base for the $V_{1}$-filter $G$ on $Y$, then $f^{-1}[C]$ is a base for the $v_{x}$-filter $\left\langle f^{-1}[G]\right\rangle$ on $X$, where $f^{-1}\left[v_{1}\right]=v_{x}$

The following result, Theorem 2.9, and Corollary 2.1 are obtained by a straightforward modification of the results in Sections 5.1.9 and 5.1.10 of [12].

THEOREM 2.9. Let $F$ and $G$ be bases for $V$ and $v_{1}$-filters (F) and $\langle G\rangle$ on $X$ and $Y$, respectively. Let $f: X \rightarrow Y$ and $*_{f}: \hat{X} \rightarrow \hat{Y}$, where ${ }^{*} f$ is the unique extension of $f$ to $\hat{X}$. Then

(i) $v_{y}$ Fil $(* f[$ Nuc $F])=\langle f[F]\rangle_{y}$,

(ii) $\operatorname{Nuc}\left(V_{y}\right.$ Fil $(* f[$ Nuc F $\left.])\right)=\operatorname{Nuc} f[F]$,

(iii) * $f^{-1}\left[\right.$ Nue G] $=$ Nue $f^{-1}[G]$.

COROLLARY 2.1. Let $* M$ be an $\alpha$-enzargement for some limit ordinal $\alpha$. Then under the hypothesis of Theorem 2.9, we have that ${ }^{*} f[$ Nuc $F]=$ Nuc $f[F]$.

If we assume that $V$ is a non-empty lattice of subsets of $X$, then 
we may use $V^{\prime}=V \cup\{\varnothing\} \cup\{X\}$ in order to topologize $\hat{X}$ by considering the set of closed sets in $\hat{X}$ to be $\left\{\right.$ Nuc $F \mid F$ is a $V^{\prime}$-filter $\}$. Indeed, since the $S$-topology on $\hat{X}$ is compact [11, p. 47] it clearly follows that $\hat{X}$ is compact in its $V^{\prime}$-topology. As a direct consequence of our previous definitions and results we have the following theorem.

THEOREM 2.10. Let $f: X \rightarrow Y$. Assume that $f^{-1}\left[\left(V^{\prime}\right)_{y}\right]=V^{\prime}$ and that $\hat{X}$ and $\hat{Y}$ carry the $V^{\prime}$ and $\left(\left(V^{\prime}\right)_{y}\right)^{\prime}$ topologies, respectively. Then

(i) the induced map *f: $\hat{X} \rightarrow \hat{Y}$ is continuous;

(ii) if $*_{M}$ is an a-enzargement for some limit ordinal $\alpha$ and if $f$ is a bijection, then ${ }^{*} f$ is a homeomorphism.

In the sequel, we will be principally concerned with $V$-ultrafilters. For this reason, the following results, even though not completely analogous to the known results for ultrafilters, are of considerable importance.

THEOREM 2.11. Let $\varnothing \in V$ and consider any $V$-ultrafizter $F$. Then

(i) $F=V \operatorname{Fil}(\{p\})=V$ Fil $(p)$ for each $p \in$ Nuc $F$;

(ii) if $F$ is fixed (that is, $\cap\{G \mid G \in F\} \neq \varnothing$ ), then there exists some $p \in X$ such that

$$
V \text { Fil }(p)=F=\{G \mid[G \in V] \wedge[\{p\} \subset G]\} ;
$$

(iii) if $F$ is free (that is, not fixed), then there exists some $q \in \hat{X}-X$ such that $\vee F i l(p)=F$ and Nuc $F \cap X=\varnothing$.

Proof. Again a simple modification of the results in Section 5.1 .5 of [12].

DEFINITION 2.2. Let $q \in \hat{X}$. If $V$ Fil $(q)$ is a $V$-ultrafilter, then we call Nuc $(V$ Fil $(q))=N V_{F}(q)$ a $V$-ultramonad. If $V$ Fil $(q)$ is free, then $N V F(q)$ is a free $V$-ultramonad.

The following important properties associated with $V$-ultramonads are obtained from the previous results in this paper or by the usual modifications of results from [11] or [12]. 
THEOREM 2.12. Let $p, q \in \hat{X}, \emptyset \in V$ and assume that $N V F(q)$, ${ }_{N} V_{F}(p)$ are $V_{\text {-ultramonads. Then }}$

(i) $N N_{F}(q), N{ }_{F}(p)$ are distinct iff $V$ Fil $(q), V$ Fil $(p)$ are distinct iff $N V F(q) \cap N V F(p)=\varnothing$ iff there exist $G, H \in V$ such that $G \cap H=\varnothing$ and $q \in \hat{G}, p \in \hat{H}$;

(ii) letting $G \in V$, we have that $G \in V$ Fil $(q)$ iff $N V F(q) \cap \hat{G} \neq \emptyset$ iff $N V F(q) \subset \hat{G}$;

(iii) letting $G \in V$, if $H \cap G \neq \varnothing$ for each $H \in V$ such that $N V_{F}(q) \cap \hat{H} \neq \varnothing$, we have that $N V_{F}(q) \cap \hat{G} \neq \varnothing$;

(iv) if $F$ is a V-filter, then Nuc $F$ is a V-ultramonad iff for each $A \in V_{-} F$, we have that Nuc $F \cap \hat{A}=\varnothing$.

The following lemma, which is proved here in its entirety, is obtained by using the enlargement $*_{M}$, the language $L$ and a suitable modification of the proof of Theorem 2.7.2 in [11].

LEMMA 2.1. Assume that we are given some non-empty $F \subset V$, an internal $\hat{Q} \subset \hat{X}$ and a map $f$ such that

(i) $f: N \rightarrow F$, where $N$ denotes the natural numbers,

(ii) $f(i) \subset f(j)$ for each $i, j \in N$ such that $j<i$,

(iii) $f(n) \neq \varnothing$ for each $n \in N$.

Then $\hat{Q} \cap(f(n))^{\wedge} \neq \varnothing$ for each $n \in N$ implies that $\hat{Q} \cap$ Nuc $f[N] \neq \varnothing$.

Proof. We have that

$$
S=\left\{q \mid\left[\left[f^{*} a p q\right] * \cap Q \neq \varnothing\right] \wedge\left[\left[f^{*} a p q\right] * \in f[N]\right] \wedge[q * \in N]\right\}
$$

is an internal subset in $\hat{N}$. Thus $S=\hat{P}$ for some $P \in * U$. Now since $f, f(n), f[N]$, and each $n \in N$ are assumed to be standard, it follows that $\hat{Q} \cap(f(n))^{\wedge}=(Q * \cap f(n))^{\wedge}=\left(Q * \cap\left(f^{*} \text { apn }\right)\right)^{\wedge} \neq \varnothing$ and $\left(f^{*}\right.$ apn $) * \in f[N]$ for each $n \in N$; which implies that $N \subset \hat{P} \subset \hat{N}$. However, $N \neq \hat{P}$ since $N$ is an external subset of $\hat{N}$. Consequently, there exists some $\nu \in \hat{N}-N$ such that $\nu \in \hat{P}$. The sentences in $L$,

$$
\begin{gathered}
\forall x \forall y[[x \in N] \wedge[y \in N] \wedge[x<y] \rightarrow[\text { fapy }] \subset[\text { fap } x]], \\
\forall x[[x \in N] \rightarrow[\sim[\text { fap } x=\emptyset]]]
\end{gathered}
$$

hold in $M$; hence in $* M$. Using the result that if $\gamma \in \hat{N}-N$, then $\gamma>n$ for each $n \in N$, we obtain $\left(f^{*} a p \nu\right)^{\wedge} \subset\left(f^{*} a p n\right)^{\wedge}=(f(n))^{\wedge}$ for each 
$n \in N$. Also $\left(f^{*} a p \nu\right)^{\wedge} \neq \varnothing$. Thus $\left(f^{*} \alpha p \nu\right)^{\wedge} \subset$ Nuc $f[N]$ and $\left(f^{*} a p \nu\right)^{\wedge} \cap \hat{Q} \neq \emptyset$. Clearly $\hat{Q} \cap$ Nuc $f[N] \neq \varnothing$.

We now use the previous results and those which the reader can easily obtain by modifying the known results in [11], [12], in order to give a nonstandard proof of a sufficient condition for a space to be Baire. We will use this result in the sequel in order to investigate a nonstardard Baire space extension of an arbitrary non-Baire space. First, we need an additional definition. The motivation for this definition comes from the well known fact that a filter $F$ converges to $p \in X$ iff Nuc $F \subset \mu(p)$, where $\mu(p)$ is the monad of Rob inson [15].

DEFINITION 2.3. A $V$-monad Nuc $F$ is said to converge on $X$ if there exists some $p \in X$ such that Nuc $F \subset \mu(p)$.

THEOREM 2.13. Let $(X, \tau)$ be a topological space. If $X$ is not a Baire space, then there exists a free converging t-ultramonat in $\hat{X}$.

Proof. Assume that $(X, \tau)$ is not Baire. Hence, there exists a nonempty $G \in \tau$ and a sequence $f:(N-\{0\}) \rightarrow P(X)$ such that $G=U\{f(n) \mid n \in N-\{0\}\}$, where $f(n) \neq \emptyset$ and int cl $f(n)=\emptyset$ for each $n \in N-\{0\}$. Define the map $h: N \rightarrow T-\{\varnothing\}$ as follows:

$$
\begin{aligned}
& \text { (i) let } h(0)=X \text {; } \\
& \text { (ii) let } h(j)=X-(U\{c 1 f(n) \mid n=1, \ldots, j\}) \text {. }
\end{aligned}
$$

Since $f(n)$ is nowhere jense in $X$, we have that $h(j)$ is an open dense set in $X$ for each $j \in N$. We observe that $h(j) \neq \emptyset$ for each $j \in N$, and that $h(i) \subset h(j)$ for each $i, j \in N$ such that $j<i$. Consider $H=\Pi\{h(j) \mid j \in N\}$. If $p \in G$, then $p \in f(k) \subset c I f(k)$ for some $k \in N-\{0\}$. Hence $p \notin h(k)$ and it follows that $G \cap H=\emptyset$. Therefore, $H$ is not dense in $X$. Let $V=\tau$ and $G(p)=\{A \mid[A \in \tau] \wedge[p \in A]\}$ for some $p \in G$. Clearly, $\mu(p)=\operatorname{Nuc} G(p)=\operatorname{Nuc}(G(p))$ and Nuc $h[N]=\operatorname{Nuc}(h[N]\rangle$. Let $E$ be the infinitesimal *element of $\langle G(p)\rangle$ which exists by Theorem 2.7. The sentence in $L$,

$$
\forall x[[x \in \tau] \wedge[\sim[x=\emptyset]] \rightarrow[\sim[x \cap h(n)=\emptyset]]]
$$

holds in $M$ for each $n \in N$; hence in $*_{M}$. Since $\langle G(p)\rangle$ is nontrivial, then $\hat{E} \neq \varnothing$, and interpreting the above sentence in $\star_{M}$ yields that $\hat{E} \cap(h(n))^{\wedge} \neq \varnothing$ for each $h(n) \in h[N]$. Lemma 2.1 implies that $\hat{E} \subset \operatorname{Nud} h[N]) \neq \varnothing$. Hence 
$\operatorname{Nuc}\langle G(p)\rangle \cap \operatorname{Nuc}\langle h[N]\rangle=\operatorname{Nuc}\langle(G(p)\rangle \cup\langle h[N]\rangle\rangle=\operatorname{Nuc} F \neq \emptyset$,

where $F=\langle\langle G(p)\rangle \cup\langle h[N]\rangle\rangle$. Thus the $\tau$-filter $F$ is non-trivial.

Therefore, there exists a $\tau$-ultramonad $N \tau F(r)$, where $r \in \hat{X}$, such that $N \tau F(r) \subset \mu(p) \cap$ Nuc $h[N]$. Now if $q \in X, q \in \mu(p)$, and $q \in(h(n))^{\wedge}$ for each $n \in N$, then $q \in \hat{H}$. This contradicts $\mu(p) \cap \hat{H}=\varnothing$. Hence $N \tau F(r) \cap X=\varnothing$. Consequently, $N \tau F(r)$ is free and obviously is converging on $X$.

\section{Baire and quasi-H-closed extensions}

In order to properly differentiate the following constructions from the usual ones, we introduce an additional definition.

DEFINITION 3.1. A topological space $Y$ will be called a nonstandard extension of a space $X$ if

(i) $X \subset Y \subset \hat{X}$ and $X \neq Y$,

(ii) $X$ is a dense subspace in $Y$.

THEOREM 3.1. If for the space $(X, \tau)$ there exists a free $\tau$-uLtramonad in $\hat{X}$, then there exists a nonstandard Baire extension $(b X, b \tau)$ of $X$.

Proof. Assume that $V=\tau$. For each $G \in \tau$, let

$N(G)=\{p \mid[p \in \hat{G}] \wedge[N \tau F(p)$ is a free $\tau-u l$ tramonad in $\hat{X}]\}$.

Define $b X=X \cup N(X)$. From the hypothesis and Theorem 2.11, we have that $X \subset b X \subset \hat{X}$ and $X \neq b X$. Also, $N(G) \subset \hat{G}-G \subset \hat{X}-X$. Now for each $G \in \tau$, let $G^{\prime}=G \cup N(G)$. Then consider $B=\left\{G^{\prime} \mid G \in \tau\right\}$.

We now show that $B$ is a base for a topology on $b X$. Let $G_{1}^{\prime}, G_{2}^{\prime} \in B$. Then $G_{1}^{\prime} \cap G_{2}^{\prime}=\left(G_{1} \cap G_{2}\right) \cup\left(N\left(G_{1}\right) \cap N\left(G_{2}\right)\right)$. If $G_{1} \cap G_{2}=\emptyset$, then $N\left(G_{1}\right) \cap N\left(G_{2}\right)=\emptyset \in B$ since $G_{1} \cap G_{2}=\varnothing$. Consequently, assume that $G_{3}=G_{1} \cap G_{2} \neq \emptyset$. Since $\hat{G}_{3}=\hat{G}_{1} \cap \hat{G}_{2}$, it is easily verified that $N\left(G_{3}\right)=N\left(G_{1}\right) \cap N\left(G_{2}\right) \cdot$ Clearly, $b X=U\left\{G^{\prime} \mid G \in \tau\right\}$. Thus we consider $B$ to be a base for a topology $b \tau$ on $b X$. It follows immediately that $X$ is a dense subspace in $b X$ and, consequently, $(b X, b \tau)$ is a nonstandard extension of $X$.

We next show that $(b X, b \tau)$ is a Baire space. Assume not. Using a 
result from McCoy's paper [13], we have that there exist sequences $\left\{U_{i}\right\}$ and $\left\{B_{i}\right\}$ of sets such that $\left\{B_{i}\right\} \subset B, U_{i} \in b \tau$ for each $i \in N$, and $B_{k+1} \subset B_{k} \subset\left(\cap\left\{U_{i} \mid i=1, \ldots, n\right\}\right), B_{k} \neq \varnothing$ for each $k \in N$. Also, $\left\{U_{i}\right\}$ is point-finite. Letting $B_{i}=G_{i} \cup N\left(G_{i}\right\}$ for each $i \in N$, it is easily shown that the sequence $\left\{G_{i}\right\}$ has the finite intersection property. Hence $\operatorname{Nuc}\left\{G_{i}\right\} \neq \varnothing$. Let $N \tau F(q)$ be a $\tau$-ultramonad contained in Nuc $\left\{G_{i}\right\}$. Then $N t F(q)$ is free since

$$
\cap\left\{G_{i} \mid i \in N\right\} \subset \cap\left\{B_{i} \mid i \in N\right\} \subset \cap\left\{U_{i} \mid i \in N\right\}=\varnothing \text {. }
$$

Consequently, $q \in \hat{X}-X$. However, we observe that since $q * \in G_{i}$ for each $i \in N$, then $q \in B_{i}$ for each $i \in N$. Thus $q \in \cap\left\{B_{i} \mid i \in N\right\}=\varnothing$. This contradiction completes the proof.

The nonstandard Baire extension $(b X, b \tau)$ has a mapping property similar to the Stone-Čech compactification. We use $V$-filter methods in order to investigate this property.

THEOREM 3.2. Let $(Z, \sigma)$ be a $T_{3}$-space and $f: X \rightarrow Z$ continuously in such a marner that $\operatorname{cl}_{Z}(f[X])=Z$ and $f[X] \neq Z$. Then the nonstandard Baire extension $(b X, b \tau)$ exists. Moreover, there exists a subspace $Y$ of $b X$ and a continuous map $h$ from $Y$ onto $Z$ such that $h \mid x=f$.

Proof. Let $V=\tau$, where $\tau$ is the topology on $X$, and assume that $p \in Z-f[X]$. Since $c l_{Z}(f[X])=Z$, then $\mu(p) \cap(f[X])^{\wedge} \neq \varnothing$. Using T-filter theory, we have that

$$
* f^{-1}[\mu(p)] \cap * f^{-1}\left[{ }^{*} f[\hat{X}]\right]=\operatorname{Nuc} f^{-1}[G(p)] \cap \hat{X} \neq \emptyset,
$$

where $G(p)=\{G \mid[G \in \tau] \wedge[p \in G]\}$. Consequently, since Nuc $f^{-1}[G(p)] \neq \emptyset$ and $f$ is continuous, then there exists a $\tau$-ultramonad $N \tau F\left(q_{p}\right) \subset{ }^{*} f^{-1}[\mu(p)]$. If $q \in X$, then $\mu(f(q)) \cap \mu(p)=\emptyset$, since $z$ is $T_{2}$. Hence, since continuity implies that $q \in \mu(q) \subset{ }^{*} f^{-1}[\mu(f(q))]$, we have that $q \notin N \tau F\left(q_{p}\right)$. Hence $N \tau F\left(q_{p}\right)$ is a free $\tau$-ultramonad and the 
nonstandard Baire extension $(b X, b \tau)$ for $X$ exists. Let

$A=\left\{N \tau F\left(q_{p}\right) \mid\left[N \tau F\left(q_{p}\right) \subset * f^{-1}[\mu(p)]\right] \wedge[p \in 2-f[X]]\right.$

$\wedge\left[N \tau F\left(q_{p}\right)\right.$ is a free $\tau$-ultramonad in $\left.\left.\hat{X}\right]\right\}$.

Define $Y=(U A) \cup X$ and consider $Y$ a subspace of $b X$.

Define $h: Y \rightarrow Z$ by $h(q)=f(q)$ for each $q \in X$ and

$h\left[N \tau F\left(q_{p}\right)\right]=p$ for each $N \tau F\left(q_{p}\right) \in A$. Observe that $A$ is a partition.

Assume that distinct $r, t \in Z-f[X]$. Since $Z$ is $T_{3}$, then there exists some $W \in \sigma$ such that $r \in W$ and $t \notin \mathrm{cl}_{Z} W$. This implies that $W \in G(r)$

and $Z-c 1 z^{W} \in G(t)$. Consequently, since $f^{-1}[W] \cap\left(2-f^{-1}\left[c 1 z^{W]}\right)=\varnothing\right.$, then $N \tau F\left(q_{p}\right) \cap N \tau F\left(q_{t}\right)=\varnothing$ and $h$ is well-defined.

Let $y \in Y$ and $W \in \sigma$ such that $h(y) \in W$. Then there exists some $W_{1} \in \sigma$ such that $h(y) \in \mathrm{cl}_{Z}\left(W_{1}\right) \subset W$. Consider

$$
\left(f^{-1}\left[W_{1}\right]\right)^{\prime}=f^{-1}\left[W_{1}\right] \cup N\left(f^{-1}\left[W_{1}\right]\right) \text {, }
$$

where $N\left(f^{-1}\left[W_{1}\right]\right)$ is defined in the proof of Theorem 3.1. Then $\left(f^{-1}\left[W_{1}\right]\right)^{\prime} \cap y=K$ is open in the topology induced on $y$. Suppose that $x \in K$. If $x \in X$, then $h(x)=f(x) \in f\left[f^{-1}\left[W_{1}\right]\right] \subset W_{1} \subset W$. If $x \in K-X$, then $x \in N \tau F\left(q_{p}\right)$ for some $p \in Z-f[X]$. If we assume that $q_{p} \notin \mathrm{cl}_{Z}\left(W_{1}\right)$, then there exists some $W^{\prime} \in \sigma$, such that $q_{p} \in W^{\prime}$ and $W_{1} \cap W^{\prime}=\emptyset$. However, this implies that $N \tau F\left(q_{p}\right)=\emptyset$ for $N \tau F\left(q_{p}\right) \subset\left(f^{-1}\left[W_{1}\right] \cap f^{-1}\left[W^{\prime}\right]\right)^{\wedge}$, a contradiction. It follows that $h[K] \subset W$. Consequently, $h$ is continuous and, obviously $h \mid X=f$. This completes the proof.

Recall that a space $X$ is quasi-H-closed [14] iff every open cover $G$ of $X$ contains a finite subset, say $\left\{G_{1}, \ldots, G_{n}\right\}$, such that $X=\left\{c l_{X}\left(G_{i}\right) \mid i=1, \ldots, n\right\}$. If $X$ is regular, then $X$ is quasi-Hclosed iff $X$ is compact. A quasi-H-closed space is also known as a 
generalized absolutely closed space [10]. Throughout the remainder of this paper, $(b X, b \tau)$ will denote the nonstandard Baire extension of a space $(X, \tau)$ when it exists.

THEOREM 3.3. If $(X, \tau)$ is not quasi-H-closed, then there exists a topology $\tau^{\prime} \supset b \tau$ on $b X$ such that $\left(b X, \tau^{\prime}\right)$ is a nonstandard quasi-Hclosed extension of $X$ and $X \in \tau^{\prime}$.

Proof. Let $V=\tau$ and construct $b X$ in the same manner as in Theorem 3.1. We observe that since $X$ is not quasi-H-closed, then there exists a non-converging $\tau$-ultramonad $N \tau F(q)$ in $\hat{X}$. Moreover, $N \tau F(q) \notin \mu(p)$ for any $p \in X$ implies that $N \tau F(q) \cap \mu(p)=\varnothing$ for each $p \in X$. Thus $N \tau F(q)$ is a free $\tau$-ultramonad in $\hat{X}$. Hence $X \subset b X$ and $X \neq b X$.

Let $B=\left\{G^{\prime} \mid G \in \tau\right\}$, where $G^{\prime}=G \cup N(G)$ and $N(G)$ is defined in the proof of Theorem 3.1. Now define $C=B \cup \tau$. Using the method indicated in the proof of Theorem 3.I, it follows easily that $C$ is a base for a topology $\tau^{\prime} \supset b \tau$ on $b X$ in which $X$ is a dense open subspace. Therefore, $\left(b X, \tau^{\prime}\right)$ is a nonstandard extension of $X$.

In order to show that $\left(b X, \tau^{\prime}\right)$ is quasi-H-closed, we need the following two results. First, a space is quasi-H-closed iff every open ultrafilter converges to an element in that space. Secondly, if $(X, \tau)$ is a dense subspace in $\left(b X, \tau^{\prime}\right)$ and $F$ is a $\tau^{\prime}$-ultrafilter on $b X$, then Nuc $(F \cap X)$ is a $\tau$-ultramonad in $\hat{X}$. In this latter case, $F \rightarrow p \in b X$ iff $\{F \cap X \mid F \in F\}=F \cap X \rightarrow p$. Let $F$ be a $\tau^{\prime}$-ultrafilter on $b X$. Assume that $\operatorname{Nuc}(F \cap X) \subset \mu(p) \subset \hat{X}$. Then $F \rightarrow p \in b X$. If $\operatorname{Nuc}(F \cap X) \notin \mu(p)$ for any $p \in X$, then $\operatorname{Nuc}(F \cap X)$ is a free $\tau$-ultramonad and $\operatorname{Nuc}(F \cap X)=N \tau F(q)$ for some $q \in b X-X$. Let $K \in \tau^{\prime}$ and assume that $q \in K$. Then there exists some $G$, $N(G) \in B$ such that $q \in G \cup N(G) \subset K$. This implies that $q \in \hat{G}$. Consequently, $(F \cap X) \cap G \neq \varnothing$ for each $F \in F$. However, for each $F \in F$, we have that $F=G_{F} \cup L_{F}$, where $G_{F} \in \tau$ and $L_{F} \subset \hat{X}-X$. Hence $G \cap G_{F} \neq \emptyset$ for each $F \in F$. Therefore, $K \cap F \neq \emptyset$ for each $F \in F$ implies that $K \in F$. It follows that $F \rightarrow q$. Thus $\left(b X, \tau^{\prime}\right)$ is quasi-H-closed and the proof is complete.

In the remaining set of theorems in this section, we discuss other nonstandard quasi-H-closed extensions and their relation to the Katétov 
extension of a Hausdorff space.

THEOREM 3.4. If $(X, \tau)$ is not quasi-H-closed, then there exists a nonstandard quasi-H-closed extension ( $h X, h \tau)$ such that $X \in h \tau$. If $X$ is also $T_{2}$, then $(h X, h \tau)$ is $T_{2}$ except for $h X-X$.

Proof. We construct the space $(h X, h \tau)$ by using a nonstandard variant of Liu's construction [10]. Let $V=\tau$ and define $H=U\{N \tau F(q) \mid N I \tau F(q)$ is a non-converging $\tau$-ultramonad in $\hat{X}\}$. For each $q \in \hat{X}$, let $G(q)=\{G \cup\{q\} \mid[G \in \tau] \wedge[N \tau F(q) \cap \hat{G} \neq \varnothing]\}$ and $B=U\{G(q) \mid q \in H\}$. Define $C=B \cup \tau$ and $h X=X \cup H$. Since $X$ is not quasi-H-closed, then $H \neq \varnothing$. Clearly, $X \subset h X \subset \hat{X}$ and $X \neq h X$. If $q \in X$, then there exists some $G \in \tau$ such that $q \in G$. If $q \in H$, then since $N \tau F(q) \cap \hat{X} \neq \varnothing$, we have that $q \in X \cup\{q\} \in B$. We know that, in general, if $N \tau F(q)$ is non-converging, then $N \tau F(q) \cap X=\emptyset$. It follows that $C$ is closed under finite intersection and is a base for a topology $h \tau$ on $h X$. Obviously, $X$ is an open dense subspace in $(h X, h \tau)$.

Let $F$ be an $h \tau$-ultrafilter on $h X$. Observe that if $\operatorname{Nuc}(F \cap X)$ is a free $\tau$-ultramonad in $\hat{X}$, then $\operatorname{Nuc}(F \cap X)=N \tau F(q)$ for some $q \in H$. If $K \in h \tau, q \in K$, then there exists some $G \cup\left\{q_{1}\right\} \in \mathcal{C}$ such that $q \in G \cup\left\{q_{1}\right\}<K$. Since $q \notin G$, then $q=q_{1}$. Thus $N \tau F(q) \cap \hat{G} \neq \emptyset$ and as in the proof of Theorem 3.3 it is easily verified that $(h X, h \tau)$ is quasi-H-closed.

Recall that a space $h X$ is said to be $T_{2}$ except for $h X-X$ iff for distinct $p, q \in h X$, not both in $h X-X$, there exist disjoint $h \tau$-open sets $K_{1}, K_{2}$ such that $p \in K_{1}$ and $q \in K_{2}$. Assume that $X$ is $T_{2}$. If distinct $p, q \in X$, then the requirement holds since $\tau \subset h \tau$. Assume that $p \in X$ and $q \in h X-X$. Since $N \tau F(q) \cap \mu(p)=\dot{\phi}$, then there exist $G, G_{p} \in \tau$ such that $p \in \hat{G}_{p}, q \in \hat{G}$, and $G_{p} \cap G=\varnothing$. Since $\hat{G}_{p} \cap \hat{G}=\varnothing$, this implies that $G_{p} \cap(G \cup\{q\})=\varnothing$, where $G \cup\{q\} \in B$. The result follows and this completes the proof.

THEOREM 3.5. Assume that $(X, \tau)$ is not quasi-H-closed. Then there exists a nonstandard quasi-H-closed extension $(k X, k \tau)$ of $X$ such that $X \in k \tau$ and with the following properties: 
(i) the space $k X$ is $T_{2}$ except for $X$;

(ii) if $X$ is $T_{2}$, then $k X$ is essentially the same as 11.107 , that is, isomorphic to [14]) the Katertov extension of $X$;

(iii) the set $h X-k X$ is infinite.

Proof. Let $V=\tau$ and

$H_{1}=\{N \tau F(q) \mid N \tau F(q)$ is a non-converging $\tau$-ultramonad in $\hat{X}\}$.

Observe that $H_{1}$ is non-empty and forms a partition for $U H_{1}$. The Axiom of Choice allows us to consider a choice set, say $H^{\prime}$, composed of one element from each element of $H_{1}$. Define $k X=X \cup H^{\prime}$. Clearly $k X \subset h X$. Let $k \tau$ be the topology induced on $k X$ by $h \tau$, where $h \tau$ is defined in the proof of Theorem 3.4. Obviously, $X$ is an open dense subspace of $(k X, k \tau)$ and $X \neq k X$.

It is easy to show that $k X$ is quasi-H-closed. Indeed, this follows in the same manner as in the proof of Theorems 3.3 and 3.4 with the additional observation that if $F$ is a $k T$-ultrafilter on $k X$ and there exists some $q \in \hat{X}-X$ such that $N \tau F(q)=\operatorname{Nuc}(F \cap X)$, then there exists some $q^{\prime} \in H^{\prime}$ such that $N \tau F\left(q^{\prime}\right)=N \tau F(q)$.

(i) Let $p \in X$ and $q \in k X-X$. In the same manner as in the proof of Theorem 3.4, it follows that $p$ and $q$ are separated by $k \tau$-open sets. Hence, we assume that we have distinct $p, q \in k X-X$. Since $N \tau F(p) \cap N \tau F(q)=\varnothing$, then there exist disjoint $G_{p}, G_{q} \in \tau$ such that $p \in \hat{G}_{p}-G_{p}$ and $q \in \hat{G}_{q}-G_{q}$. The result follows easily.

(ii) Recall that $k X$ is essentially the same as (that is, isomorphic to) the Katètov extension of $X$ iff there exists a homeomorphism from $k X$ onto the Katètov extension of $X$ which restricted to $X$ is the identity. We use the criterion of Liu's [10]. If $X$ is $T_{2}$, then a $T_{2}$ extension $Y$ of $X$ is essentially the same as Katétov's extension if all sets of the form $G \cup\{q\}$, where $G \in \tau$ and $q \in c l_{Y}(G)-X$, are open in $Y$ and $X$ is open in $Y$. Clearly $(i)$ implies that $k X$ is $T_{2}$. Let $G \in \tau$ and $q \in \mathrm{cl}_{k X}(G)-X \subset k X-X$. Assume that $q \in G_{1} \cup\left\{q_{1}\right\} \in B$, where $B$ is 
defined in the proof of Theorem 3.4. Then $q=q_{I}$. However, $\left(G_{1} \cup\{q\}\right) \cap G \neq \emptyset$ implies that $G_{1} \cap G \neq \emptyset$. Hence $N \tau F(q) \subset \hat{G}$. Consequently, $q \in \hat{G}$ implies that $G \cup\{q\} \in B$ and the result follows.

(iii) There exists some $q \in \hat{X}-X$ such that $N \tau F(q) \cap X=\emptyset$ and $N \tau F(q) \neq \emptyset$. Since Nuc Fil $(q) \subset N \tau F(q)$, the conclusion is obvious since Nuc $F i l(q)$ is an infinite set.

THEOREM 3.6. Asswe that $(X, \tau)$ is not quasi-H-closed and that $(Y, \gamma)$ is any subspace of $h X$ such that $k X \subset Y$.

(i) Then $(Y, \gamma)$ is a dense open subspace.

(ii) AZso, $(Y, \gamma)$ is a nonstandard quasi-H-closed extension of $X$ such that $X \in \gamma$ and if $k X \neq Y$, then $Y$ is not $T_{2}$ except for $X$.

Proof. (i) The subspace $Y$ is obviously dense in $h X$. Let $q \in Y$. If $q \in X$, then there exists some $G \in \tau$ such that $q \in G \subset Y$. If $q \notin X$, then $q \in H$, where $H$ is defined in the proof of Theorem 3.4. However, $H^{\prime}$ defined in Theorem 3.5 is a choice set for the partition $H_{1}$ of $H$. We observe that there exists one, and only one, $N_{\tau} F\left(q_{1}\right) \in H_{1}$ such that $q \in N \tau F\left(q_{1}\right)$. For some $G \in \tau$, it follows that $G \cup\{q\} \in h \tau$ and $G \cup\{q\} \subset Y$. Thus $Y$ is open in $h X$.

(ii) A proof similar to that which shows that $k X$ and $h X$ are quasi-H-closed also shows that $(Y, \gamma)$ is quasi-H-closed. Clearly $X \in Y$.

Now if $k X \neq Y$, then it is clear that there exist distinct $p, q \in Y$ such that $N \tau F(q)=N \tau F(p)$. Consequently, if $G \in \tau$, then $p \in \hat{G}$ iff $q \in \hat{G}$. This implies that $Y$ is not $T_{2}$ except for $X$ and the proof is complete.

The final result in this section shows that the space $k X$ constructed in Theorem 3.5 is the smallest nonstandard quasi-H-closed extension in a very natural sense.

THEOREM 3.7. Assume that $(X, \tau)$ is not quasi-H-closed. If $X \subset Y \subset k X$ and $Y$ is a proper subspace, then $Y$ is not quasi-H-closed.

Proof. Assume that $q \in k X-Y$. Then $N T F(q)$ is a free non- 
converging $\tau$-ultramonad. Let $F=\{K \mid[K \in k \tau] \wedge[K \cap X \in \tau$ Fil $(q)]\}$. It is known that $F$ is a kT-ultrafilter on $k X$. Conversely, $F \cap X$ is a $\tau$-ultrafilter on $X$ such that $F \cap X=\tau$ Fil $(q)$. Clearly $F \rightarrow q$. Now if $q^{\prime} \neq q$ and $q^{\prime} \in k X-X$, then since $k X$ is $T_{2}$ except for $X$, we have that $F+q^{\prime}$. We know that $F \cap Y \rightarrow p$ for some $p \in Y$ iff $F \rightarrow p$. Thus $F \cap Y \rightarrow q$ and only $q \in k X$. However, $q \notin Y$ and the result follows.

\section{Wallman type compactifications}

In this section, we construct nonstandard Wallman type compactifications which are not necessarily Hausdorff. We will do this by letting $V=B$, where $B$ is a normal base for the space $X$ as originally defined by Frink [5] with the $T_{1}$ requirement deleted. Unless otherwise indicated, the space $(X, \tau)$ will be non-compact completely regular and not necessarily $T_{1}$. Observe, that a normal separating ring of closed sets in the sense of Steiner [19] is a normal base and conversely.

THEOREM 4.1. Each normal base $\beta$ for $(X, \tau)$ determines nonstandard normal compactifications $H(X, \beta)$ and $\omega(X, \beta)_{S}$ such that $X \subset \omega(X, \beta)_{S} \subset H(X, \beta) \subset \hat{X}$.

Proof. Assume that $B$ is a normal base. Since $(X, \tau)$ is noncompact, then there exists a non-empty $\left\{B_{\lambda} \mid \lambda \in \Lambda\right\}=F \subset \beta$ with the finite intersection property and such that $\cap F=\emptyset$. Since $* M$ is an enlargement, it follows that Nuc $F \neq \varnothing$. Thus, for some $q \in$ Nuc $F$, we have that $N \beta F(q)$ is a free $\beta$-ultramonad. We now construct $H(X, \beta)$. For each $B \in \beta$, let

$$
F(B)=\{q \mid[q \in \hat{B}] \wedge[N \beta F(q) \text { is a free } \beta \text {-ultramonad in } \hat{X}]\} \text {. }
$$

Define $H(X, \beta)=X \cup F(X)$. Clearly, $X \subset H(X, \beta) \subset \hat{X}$ and $X \neq H(X, \beta)$.

In order to obtain a topology for $H(X, \beta)$, let $B^{\prime}=B \cup F(B)$ for each $B \in \beta$. Consider $\Gamma=\left\{B^{\prime} \mid B \in \beta\right\}$. If $B^{\prime}, C^{\prime} \in \Gamma$, then $B^{\prime} \cup C^{\prime}=(B \cup C) \cup(F(B) \cup F(C))$. However, $B \cup C=D \in \beta$ and it follows easily that $F(D)=F(B) \cup F(C)$, since $\hat{B} \cup \hat{C}=\hat{D}$. Notice that $\emptyset, H(X, B) \in \Gamma$. Hence, we consider $\Gamma$ a base for the closed subsets in 
$H(X, B)$. Obviously $X$ is a subspace in $H(X, B)$ since $F(B) \cap X=\varnothing$ for each $B \in \beta$. Considering any $B \cup F(B) \in \Gamma$ such that $X \subset B \cup F(B)$, we have that $X=B$. Consequently, $X$ is dense in $H(X, \beta)$.

We now show that $H(X, \beta)$ is compact. Let $\left\{B_{\phi}^{\prime} \mid \phi \in \Phi\right\}$ be a set of basic closed sets in $H(X, \beta)$ with the finite intersection property. Assume that $\left\{B_{i}^{\prime} \mid i=1, \ldots, n\right\} \subset\left\{B_{\phi}^{\prime} \mid \phi \in \Phi\right\}$ for some $n>1$.

Clearly

$$
\begin{aligned}
n\left\{B_{i}^{\prime} \mid i\right. & =1, \ldots, n\}= \\
& =\left(\cap\left\{B_{i} \mid i=1, \ldots, n\right\}\right) \cup\left(\cap\left\{F\left(B_{i}\right) \mid i=1, \ldots, n\right\}\right)=B \cup F(B),
\end{aligned}
$$

where $B=\cap\left\{B_{i} \mid i=1, \ldots, n\right\} \in \beta$. Now $B \cup F(B) \neq \emptyset$. If $B=\varnothing$, then $F(B)=\varnothing$. Consequently $B \neq \varnothing$ implies that

$\left\{B_{\phi} \mid[\phi \in \Phi] \wedge\left[B_{\phi}^{\prime}=B_{\phi} \cup F\left(B_{\phi}\right)\right]\right\}=F^{\prime}$ has the finite intersection

property. Thus, Nuc $F^{\prime} \neq \varnothing$. If there exists some $p \in X$ such that $p \in$ Nuc $F^{\prime}$, then $p \in B_{\phi}$ for each $\phi \in \Phi$. On the other hand, assuming that there does not exist a $p \in X$ such that $p \in$ Nuc $F^{\prime}$, then there exists some $q \in \hat{X}-X$ such that the free $\beta$-ultramonad $N \beta F(q) \subset \mathbb{N u c} F^{\prime}$. Since $q \in \hat{B}_{\phi}$ for each $\phi \in \Phi$, then this implit that $r<F(R)$ for each $\phi \in \Phi$. Consequently, $\cap\left\{B_{\phi}^{\prime} \mid \phi \in \Phi\right\} \neq \varnothing$ and $H(X, B)$ is compact.

We now show that $\Gamma$ is a separating family of closed sets in the sense of Steiner [18]. Let $K$ be any non-empty closed subset in $H(X, \beta)$ and assume that $q \in H(X, B)-K$. We first assume that $q \in X$. Since $X$ is a subspace, then $K \cap X$ is closed in $X$. Since $\Gamma$ is a base for the closed sets in $H(X, \beta)$, it follows that $K \subset B \cup F(B)$ and $q$ k. $B \cup F(B)$ for some $B \cup F(B) \in \Gamma$. However, $B$ is a separating family implies that there exist $C, D \in B$ such that $C \cap D=\varnothing, K \cap X \subset B \subset C$, and $q \in D$. Clearly, $F(C) \cap F(D)=\emptyset$ and $F(B) \subset F(C)$ imply that $K \subset C \cup F(C)$, $q \in D \cup F(D)$, and $(C \cup F(C)) \cap(D \cup F(D))=\varnothing$. On the other hand, we assume that $q \in \hat{X}-X$. Thus, $q \in F(B)$ for some $B \in \beta$. In the same manner as above, we easily show that there exist elements of $\Gamma$ which separate $K$ and $q$. Consequently, $\Gamma$ is a separating family of closed sets.

In order to show that $\Gamma$ is a normal separating family [17], we 
assume that $B_{1} \cup F\left(B_{1}\right)=B_{1}^{\prime}, B_{2} \cup F\left(B_{2}\right)=B_{2}^{\prime}$ and $B_{1}^{\prime} \cap B_{2}^{\prime}=\varnothing$. The base $\beta$ being normal implies that there exist $B_{3}, B_{4} \in \beta$ such that $B_{1} \subset B_{3}, B_{2} \subset B_{4}, B_{1} \cap B_{4}=B_{2} \cap B_{3}=\varnothing$, and $B_{3} \cup B_{4}=X$. If we consider the sets $B_{i} \cup F\left(B_{i}\right), i=1,2,3,4$, then it is easily verified that $\Gamma$ is normal. Consequently, $\Gamma$ is a normal separating family of closed sets and Steiner's result in [18] implies that $H(X, \beta)$ is a completely regular space. Hence $H(X, \beta)$ is a normal compactification of $X$.

We now construct $\omega(X, \beta)_{S}$. Obviously $\{N B F(q) \mid q \in F(X)\}$ partitions $F(X)$. Let $S(\beta)$ be the set of all choice sets determined by $\{N \beta F(q) \mid q \in F(X)\}$ and $S \in S(\beta)$. Define $\omega(X, \beta)_{S}=X \cup S$ and let $\omega(X, \beta)_{S}$ carry the topology induced by $\Gamma$. Hence, if $B \in \beta$ and $q \in \hat{B}$ such that $N B F(q)$ is a free $\beta$-ultramonad in $\hat{X}$, then there exists one, and only one, element, say $p \in S$, such that $N \beta F(q)=N \beta F(p)$. It is also clear that for each $B \in \beta$ such that $F(B) \neq \varnothing$ the set $S \cap F(B)$ is a choice set for the partition $\{N \beta F(q) \mid q \in F(B)\}$. Observe that the closed base for $\omega(X, B)_{S}$ induced by $\Gamma$ is of the form $\{B \cup(S \cap F(B)) \mid B \in \beta\}$. To show that $\omega(X, B)_{S}$ is a normal compactification of $X$, it is sufficient to show that $\omega(X, B)_{S}$ is a compact subspace of $H(X, \beta)$. However, this follows from a simple modification of the above proof that $H(X, \beta)$ is compact and this completes the proof.

Throughout the remainder of this paper, we shall let $S(B)$ be the set of all choice sets determined by the partition $\{N \beta F(q) \mid q \in F(X)\}$, where $\beta$ is a normal base for $X$. Also, $\omega(X, \beta)_{S}$ for some $S \in S(\beta)$ and $H(X, B)$ will denote the normal compactifications of $X$ constructed in Theorem 4.1. In [4], D'Aristotle constructs for each normal base $\beta$ a normal compactification $\chi^{(\beta)}$. Our next theorems show the relations between the compactifications $\omega(X, \beta)_{S}, H(X, \beta)$, and $\chi^{(\beta)}$. In particular, they show that $\omega(X, \beta)_{S}$ is homeomorphic $(\simeq)$ to $\chi^{(\beta)}$ and

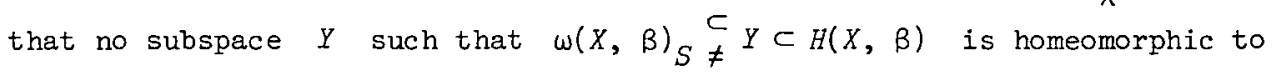


$X^{(\beta)}$ even though all such $Y$ are normal compactifications of $X$.

THEOREM 4.3. The compactification $\omega(X, B)_{S} \simeq \chi^{(B)}$.

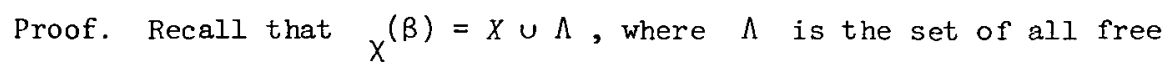
$\beta$-ultrafilters on $X$. Define the map $\phi: x^{(\beta) \rightarrow \omega(X, \beta)} S$ in the following manner. For each $p \in X$, let $\phi(p)=p$. For $\lambda \in \Lambda$, let $\phi(\lambda)=S \cap \operatorname{Nuc}(\lambda)=q \in \operatorname{Nuc}(\lambda)$. Clearly, $\phi$ is a bijection. Since for each $B \in B, \phi$ maps the closed base elements $B \cup\{\lambda \mid[\lambda \in \Lambda] \wedge[B \in \lambda]\}$ of $\chi^{(B)}$ onto $B \cup(S \cap F(B))$, then $\phi$ is a homeomorphism.

COROLLARY 4.1. If $(X, \tau)$ is a Tychonoff space, then $\omega(X, \beta)_{S} \simeq \omega(X, \beta)$, where $\omega(X, \beta)$ is the $T_{2}$ Wallman compactification of $X$ in the sense of Frink [5].

THEOREM 4.4. Assume that $Y$ is a subspace of $H(X, B)$ such that $\omega(X, B)_{S} \varsubsetneqq Y$. Then $Y$ is a non-T $T_{0}$ normal compactification of $X$.

Proof. Since it is easily verified that $Y$ is a normal compactification of $X$, all we need to show is that $Y$ is not $T_{0}$. Clearly there exist distinct $p, q \in Y-X$ such that $N \beta F(q)=N \beta F(p)$. Now for any $B \cup F(B) \in \Gamma$, we have that $p \in F(B)$ iff $q \in F(B)$. Hence $Y$ is not $T_{0}$.

If we assume that a free $\beta$-uItramonad $N \beta F(q)$ is a finite set, then there exists a non-empty finite infinitesimal *element $E$ such that $\hat{E} \subset N \beta F(q)$. However $E * \in \beta \mathrm{FiI}(q)$ implies by transfer that there exists a non-empty finite element of $\beta$ which is an element of the free $\beta$-ultrafilter $\beta$ Fil $(q)$. This contradiction implies that $N \beta F(q)$ is an infinite set. Consequently, for each normal base $\beta$ for $X$ there exist infinitely many distinct yet homeomorphic compactifications $\omega(X, \beta)_{S}$ which are all homeomorphic to the $T_{2}$ Wallman compactification $\omega(X, B)$ if $X$ is $T_{1}$. For this reason, the following result is not without interest.

THEOREM 4.5. Assume that $(X, \tau)$ is Tychonoff and $B, \gamma$ are normal 
bases such that $\beta \subset \gamma$. Then $\omega(X, \beta) \simeq \omega(X, \gamma)$ iff $S(Y) \subset S(\beta)$ and for each $S \in S(\gamma), \omega(X, \beta)_{S}=\omega(X, \gamma)_{S}$ as spaces.

Proof. Sufficiency is apparent. Hence, let $N \gamma F(q) \subset H(X, \gamma)$ and consider the $\beta$-monad $N \beta F(q)$. We know that $N \gamma F(q)$ is a $\gamma$-ultramonad and, since $\beta \subset \gamma$, it follows that $N \gamma F(q) \subset N \beta F(q)$.

We need to show that $N \beta F(q)$ is a free $\beta$-ultramonad. Let $B \in B$ such that $q \notin \hat{B}$. Then since $B \subset \gamma$ there must exist some $C \in \gamma$ such that $q \in N Y F(q) \subset \hat{C}$ and $B \cap C=\emptyset$ for $N \gamma F(q)$ is a $\gamma$-ultramonad. Using Steiner's Theorem 7 in [19], which implies that $\beta$ separates $\gamma$, we have that there exist $B_{1}, B_{2} \in B$ such that $B \subset B_{I}, C \subset B_{2}$, and $B_{1} \cap B_{2}=\emptyset$. Since $q \in \hat{B}_{2}$ implies that $B_{2} \in B$ Fil $(q)$, we have that $N B F(q)$ is a $B$-ultramonad for $B \cap B_{2}=\varnothing$. Next we show that $N B F(q)$ is free. Since $N \gamma F(q)$ is a free $\gamma$-ultramonad, we know that, for each $p \in X$, there exists some $C_{p} \in \gamma$ such that $N \gamma F(q) \subset \hat{C}_{p}$ and $p \leqslant C_{p}$. However there exist $B_{3}, B_{4} \in \beta$ such that $p \in B_{3}, C_{p} \subset B_{4}$, and $B_{1} \cap B_{4}=\varnothing$ since $B$ separates points and closed sets. Clearly this implies that $N B F(q) \subset \hat{B}_{4}$ since $N \beta F(q)$ is a $\beta$-ultramonad. Hence $p \notin N B F(q)$ implies that $N B F(q)$ is free.

Assume that $N B F\left(q^{\prime}\right) \subset H(X, \beta)$. Clearly $N B F\left(q^{\prime}\right)$ is a free $\gamma$-monad since $N B F\left(q^{\prime}\right)$ is a free $\beta$-ultramonad and $\beta \subset \gamma$. Thus there exists a free $\gamma$-ultramonad $N \gamma F\left(q^{\prime \prime}\right) \subset N \gamma F\left(q^{\prime}\right)$. Therefore $N \gamma F\left(q^{\prime \prime}\right) \subset N \beta F\left(q^{\prime \prime}\right) \subset H(X, \beta)$. However, $N \beta F\left(q^{\prime \prime}\right)=N \beta F\left(q^{\prime}\right)$. Consequently, if $N \beta F\left(q^{\prime}\right) \subset H(X, \beta)$, there there exists some $N \gamma F\left(q^{\prime \prime}\right) \subset H(X, \gamma)$ such that $N Y F\left(q^{\prime \prime}\right) \subset N B F\left(q^{\prime}\right) \subset H(X, \beta)$. Assume that there exists some $\operatorname{N\gamma F}\left(q_{1}\right) \subset H(X, \gamma)$ such that $\operatorname{N\gamma F}\left(q_{1}\right) \subset N B F\left(q^{\prime}\right)$ and $N \gamma F\left(q_{1}\right) \neq N \gamma F\left(q^{\prime \prime}\right)$. Then there exist $c_{1}, c_{2} \in \gamma$ such that $q_{1} \in \hat{C}_{1}, q^{\prime} \in \hat{C}_{2}$, and $C_{1} \cap C_{2}=\emptyset$. Since $\beta$ separates $\gamma$, it now easily follows that $N B F\left(q_{1}\right) \subset N B F\left(q^{\prime \prime}\right) \subset H(X, \beta)$ and $N B F\left(q_{1}\right) \cap N B F(q)=\varnothing$. However $N \beta F\left(q_{1}\right)=N \beta F\left(q^{\prime \prime}\right)=N \beta F\left(q^{\prime}\right)$, a contradiction. Consequently, there exists

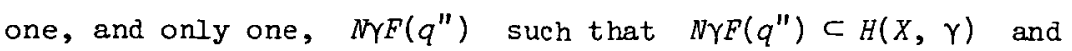
$N \gamma F\left(q^{\prime \prime}\right) \subset N B F\left(q^{\prime}\right)$. 
It is clear from the above results that $S(\gamma) \subset S(\beta)$. Hence $\omega(X, \beta)_{S}=\omega(X, \gamma)_{S}$ as sets for each $S \in S(\gamma)$. The closed base $\{B \cup\{S \cap F(B)) \mid B \in \beta\}$ for $\omega(X, \beta)_{S}$ is a subset of the closed base $\{C \cup\{S \cap F(C)) \mid C \in \gamma\}$ for $\omega(X, \gamma)_{S}$. Since the topologies for $\omega(X, \beta)_{S}$ and $\omega(X, \gamma)_{S}$ are $T_{2}$ compact, it follows that $\omega(X, B)_{S}=\omega(X, \gamma)_{S}$ as spaces. This completes the proof.

\section{References}

[1] R.A. Alo and H.L. Shapiro, "A note on compactifications and seminormal spaces", J. Austral. Math. Soc. 8 (1968), 102-108.

[2] Richard A. Alò and Harvey L. Shapiro, "Wallman compact and realcompact spaces", Contributions to extension theory and topological structures, 9-14 (Proc. Sympos., Berlin, 1967. Deutscher Verlag der Wissenschaften, Berlin; Academic Press, New York; 1969).

[3] Charles M. Biles, "Wallman-type compactifications", Proc. Amer. Math. Soc. 25 (1970), 363-368.

[4] Anthony J. D'Aristotle, "Completely regular compactifications", Frond. Math. 71 (1971), 139-145.

[5] Orrin Frink, "Compactifications and semi-normal spaces", Amer. $J$. Math. 86 (1964), 602-607.

[6] Leonard Gillman and Meyer Jerison, Rings of continuous functions (Van Nostrand, Princeton, New Jersey; Toronto; London; New York; 1960).

[7] Robert A. Herrmann, "V-filters", paper presented to the seventy-ninth ainual meeting Amer. Math. Soc., Dallas, Texas, January, 1973 (see also: Abstract No. 701-54-14, Notices Amer. Math. Soc. 20 (1973), A-173).

[8] R.A. Herrmain, "H(i) semiregular, $U(i)$ and $R(i)$ extensions", submitted. 
[9] M. Katētov, "Über H-abgeschlossene und bikompakte Räume", Časopis Pěst. Mat. Fys. 69 (1939), 36-49.

[10] Chen-Tung Liu, "Absolutely closed spaces", Trans. Amer. Math. Soc. 130 (1968), 86-104.

[11] W.A.J. Luxemburg, "A general theory of monads", Applications of model theory to algebra, analysis, and probability, 18-86 (Internat. Sympos., Pasadena, California, 1967. Holt, Rinehard and Winston, New York, Chicago, San Francisco, Atlanta, Dallas, Montreal, Toronto, London, Sydney, 1969).

[12] Moshé Machover, Joram Hirschfeld, Lectures on non-standard analysis (Lecture Notes in Mathematics, 94. Springer-Verlag, Berlin, Heidelberg, New York, 1969).

[13] R.A. McCoy, "A Baire space extension", Proc. Amer. Math. Soc. 33 (1972), 199-202.

[14] Jack Porter and John Thomas, "On H-closed and minimal Hausdorff spaces", Trans. Amer. Math. Soc. 138 (1969), 159-170.

[15] Abraham Robinson, Non-standard analysis (Studies in Logic and the Foundations of Mathematics. North-Holland, Amsterdam, 1966).

[16] P. Samuel, "Ultrafilters and compactifications of uniform spaces", Trans. Amer. Math. Soc. 64 (1948), 100-132.

[17] A.K. Steiner and E.F. Steiner, "Wallman and Z-compactifications", Duke Math. J. 35 (1968), 269-275.

[18] E.F. Steiner, "Normal families and completely regular spaces", Duke Math. J. 33 (1966), 743-745.

[19] E.F. Steiner, "Wallman spaces and compactifications", Frond. Math. 61 $(1967 / 68), 295-304$.

Department of Mathematics,

US Naval Academy,

Annapolis,

Maryland,

USA. 